\title{
Estudios con fitoderivados en patología experimental: tendencias y recomendaciones
}

\author{
Studies with phytoderivatives in experimental pathology: tendencies and
}

recommendations

\author{
Yesenia M. Huerta-Collado ${ }^{1,2, a}$, Miluska O. Mejia ${ }^{1,2, a}$, Liz Veramendi-Espinoza ${ }^{1,2, a}$, \\ Juan José Montenegro-Idrogo $0^{1,2, b}$ \\ Facultad de Medicina, Universidad Nacional Mayor de San Marcos. Lima, Perú. \\ Sociedad Cientifica San Fernando- Universidad Nacional Mayor de San Marcos. Lima, Perú. \\ ${ }^{a}$ Estudiante de Medicina; ${ }^{b}$ Médico cirujano.
}

\section{An Fac med. 2014;75(2):205-6 / doi: http://dx.doi.org/10.15381/anales.v75i2.8391}

\section{SEÑOR EDITOR}

La universidad es un ente que permite la generación de conocimientos a través de la investigación, la cual responde a necesidades regionales y globales que ayudarán a la toma de decisiones e instauración de políticas ${ }^{(1)}$. Para ello, las universidades se organizan mediante centros de investigación, los cuales tienen líneas de investigación defini- das; de este modo las investigaciones se hacen sostenibles y contextuales.

Cabe destacar que a nivel nacional se ha evidenciado que hay interés creciente en cuanto a la línea de investigación sobre plantas medicinales, siendo la Universidad Nacional Mayor de San Marcos (UNMSM) una de las que más publicaciones en revistas biomédicas nacionales tiene en esta línea ${ }^{(2)}$. Así

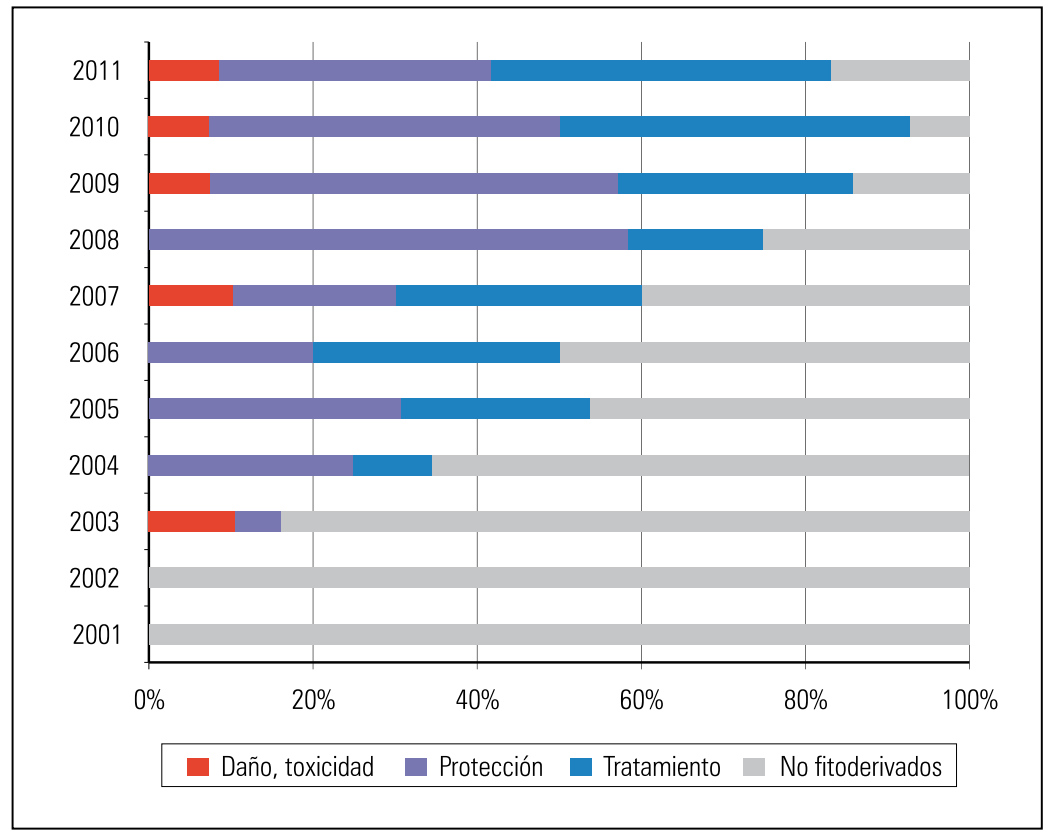

Gráfica 1. Porcentaje de investigaciones con fitoderivados según efecto del estudio, y según año.
Tabla 1. Fitoespecies más utilizadas en investigaciones de estudiantes de medicina de Patología Experimental de la UNMSM, en 11 años.

\begin{tabular}{lcc} 
Fitoespecie & $\mathrm{N}^{0}$ trabajos & $(\%)$ \\
\hline Erithroxylon coca & 6 & $(7,9)$ \\
Curcuma longa & 4 & $(5,3)$ \\
Lepidemium meyenii & 3 & $(3,9)$ \\
Myrciaria dubia & 3 & $(3,9)$ \\
Phaseolus vulgaris L. & 3 & $(3,9)$ \\
Physalis peruviana & 3 & $(3,9)$ \\
Plukenetia volubilis & 3 & $(3,9)$ \\
Capsicum pubescens & 2 & $(2,6)$ \\
Coffea arabica & 2 & $(2,6)$ \\
Gentianella alborosea & 2 & $(2,6)$ \\
Peumus boldus & 2 & $(2,6)$ \\
Solanum tuberosum & 2 & $(2,6)$ \\
Tabebula serratifolia & 2 & $(2,6)$ \\
Tropaeolum tuberosum & 2 & $(2,6)$ \\
Zea mays I. & 2 & $(2,6)$ \\
Otros & 35 & $(18,0)$ \\
\hline
\end{tabular}

mismo se manifiesta que la producción en esta área no es ajena a estudiantes de medicina ${ }^{(3)}$. Es así que en el pregrado de la Escuela de Medicina de la UNMSM existen cursos que en su plan curricular exigen la elaboración de trabajos de investigación de tipo experimental, como es el caso del curso Patología Experimental, en cuyas jornadas se observa un gran número de trabajos que estudian fitoderivados. 
Debido a ello, se ha recopilado los trabajos de estudiantes de medicina de dicho curso (uno de los cursos de ciencias básicas de la Facultad de Medicina que continuamente produce estudios de experimentación con animales) desarrollados en 11 años. Se observó una tendencia creciente de trabajos en los que se usa fitoderivados para evidenciar su toxicidad, protección o como tratamiento (gráfica 1). De 183 informes de trabajos completados desde 2001 al 2011, 76 investigaciones (42\%) correspondieron a estudios sobre fitoderivados. También se evidenció que el número de estudios sobre un determinado fitoderivado se limitó a una o dos investigaciones (tabla 1).
Consideramos que el curso de Patología Experimental es un espacio que constantemente genera investigaciones como parte del su plan curricular y hay un mayor interés en el estudio de fitoderivados a lo largo del tiempo. Sin embargo, se observa que un determinado fitoderivado no es estudiado continuamente, no permitiendo así un mejor entendimiento sobre sus propiedades o, si lo hacen, es de manera inconexa. Por ello, se sugiere establecer áreas temáticas en relación a los productos y los efectos que se considere más relevantes de estudiar. Plantear así estudios dirigidos en determinados temas permitirá que estas investigaciones sean mejor elaboradas y el aporte sea mayor en relación a estos productos y sus efectos de interés en biomedicina.

\section{REFERENCIAS BIBLIOGRÁFICAS}

1. Sedlacek S. The role of universities in fostering sustainable development at the regional level. J Clean Prod. 2013;48:74-84. doi: http://dx.doi. org/10.1016/j.jclepro.2013.01.029

2. Pamo-Reyna OG. Características de los trabajos publicados sobre las propiedades de las plantas en revistas médicas peruanas. Rev peru med exp salud publica. 2009;26 (3):314-23.

3. Arroyo-Hernández $\mathrm{CH}$, Angulo-Bazán Y. Publicaciones estudiantiles sobre propiedades medicinales de las plantas en Perú. Rev peru med exp salud publica. 2009;26(4):585-6.

Los autores declaran no existir conflictos de interés.

Autor corresponsal:

Yesenia M. Huerta-Collado

Correo electrónico:ymhuertac@gmail.com 